Research in Astron. Astrophys. 20xx Vol. xx No. xx, 000-000

http://www.raa-journal.org http://www.iop.org/journals/raa

$\boldsymbol{R}$ esearch in

Astronomy and

Astrophysics

\title{
Dust growth in protoplanetary disks - a comprehensive experimental/theoretical approach
}

\author{
J. Blum \\ Institut für Geophysik und extraterrestrische Physik, University of Braunschweig, Mendelssohnstr. 3, \\ 38106 Braunschweig, Germany;j.blum@tu-braunschweig.de \\ Received [year] [month] [day]; accepted [year] [month] [day]
}

\begin{abstract}
More than a decade of dedicated experimental work on the collisional physics of protoplanetary dust has brought us to a point at which the growth of dust aggregates can for the first time - be self-consistently and reliably modelled. In this article, the emergent collision model for protoplanetery dust aggregates (Güttler et al., 2010) as well as the numerical model for the evolution of dust aggregates in protoplanetary disks (Zsom et al., 2010) are reviewed. It turns out that, after a brief period of rapid collisional growth of fluffy dust aggregates to sizes of a few centimeters, the protoplanetary dust particles are subject to bouncing collisions, in which their porosity is considerably decreased. The model results also show that low-velocity fragmentation can reduce the final mass of the dust aggregates but that it does not trigger a new growth mode as discussed previously. According to the current stage of our model, the direct formation of kilometer-sized planetesimals by collisional sticking seems impossible so that collective effects, such as the streaming instability and the gravitational instability in dust-enhanced regions of the protoplanetary disk, are the best candidates for the processes leading to planetesimals.
\end{abstract}

Key words: planetary systems: protoplanetary disks, planetary systems: formation, methods: laboratory, methods: numerical

\section{INTRODUCTION AND MOTIVATION}

The 'standard' paradigm of planet formation comprises a two-stage process: (1) When the particles are small, i.e. when we speak of 'dust', growth is supposed to be by coagulation, i.e. dust particles stick to one another due to non-gravitational forces, e.g. surface adhesion (van der Waals force). (2) For much larger particles, i.e. for 'planetesimals', the further growth is based on accretion, i.e. mutual gravitational attraction of the colliding bodies.

As the gravitational potential of an individual body increases with its mass, stage (2) requires planetesimal sizes of at least $\sim 1 \mathrm{~km}$, i.e. escape velocities of the order of $1 \mathrm{~m} \mathrm{~s}^{-1}$, before accretion in mutual collisions becomes effective. Thus, we are faced with the problem to explain dust growth in protoplanetary disks (PPDs) due to 'sticking collisions' for a range of dust masses spanning 27 orders of magnitude, i.e. dust sizes ranging from $\sim 1 \mu \mathrm{m}$ to $\sim 1 \mathrm{~km}$.

To better assess the scenario of dust growth in PPDs, we will first look at the prerequisites for collisional evolution, i.e. the causes of mutual collisions among the dust grains. Current models of PPDs (see, e.g., Dullemond et al. (2007)) favor geometrically thin (but optically thick) flared disk structures with the gas pressure and temperature decreasing radially outward and a modest degree of MRI-driven turbulence in the gas. In such a scenario, the embedded dust particles undergo a variety of random 
and systematic motions, which lead to frequent collisions among them (Weidenschilling, 1977). Very small particles (sizes $\lesssim 100 \mu \mathrm{m}$ ) are mostly affected by Brownian motion, which leads to collisions at extremely low velocities, i.e. $v \lesssim 10^{-3} \mathrm{~m} \mathrm{~s}^{-1}$. Larger grains are subject to systematic motion with respect to the nebular gas: particles outside the midplane of the PPD sediment towards the midplane, due to the vertical component of the gravitational attraction of the central star; in addition to that, dust particles spiral radially inward, owing to their friction with the slower rotating (pressure-supported) gas disk. Both drift velocities increase with increasing mass-to-surface ratio of the dust particles so that large dust aggregates catch up with small ones. Turbulence in the gas also causes dust particles to collide with one another, also for dust aggregates with identical masses. Weidenschilling \& Cuzzi (1993) derived collision velocities for all combinations of dust sizes, starting from small grains with sizes of $1 \mu \mathrm{m}$ all the way to the smallest planetesimals. Besides the above-mentioned regime, where Brownian motion dominates the collisional evolution of the dust grains and in which the collision velocity decreases with the mass of the aggregates, the collision velocity typically increases with increasing size of the dust grains up to meter-sized bodies, after which collision speeds stay rather constant at values around $v \approx 50 \mathrm{~m} \mathrm{~s}^{-1}$. An improved model of turbulence-induced collision velocities was published by Ormel \& Cuzzi (2007) who give closed-form solutions for all particle-size combinations. It should be mentioned that all relative velocities (with the exception of those caused by Brownian motion) are a function of gas density and strength of turbulence, usually characterized by an $\alpha$ value.

It will be shown later (see Sect. 3) that a critical velocity in the collisional evolution is at $v \approx 1 \mathrm{~m} \mathrm{~s}^{-1}$, above which dust aggregates tend to fragment in collisions. In the minimum-mass solar nebula (MMSN) model with $\alpha \approx 10^{-4}$, assumed by Weidenschilling \& Cuzzi (1993), this collision velocity is reached for cm-sized dust particles; lower turbulence strength of $\alpha=10^{-5}$ increases the size at which the fragmentation velocity is reached to decimeters (see Weidling et al. (2009)). Other PPD models, having different gas pressures, gas temperatures and pressure gradients, exhibit a similar velocity systematics but can reach the critical velocity for fragmentation for very different dust-aggregate sizes (see, e.g., Weidling et al. (2009); Zsom et al. (2010)).

Astronomical observations of PPDs at various wavelengths yield strong indications for grain growth (Natta et al., 2007). Unfortunately, aggregate sizes in excess of a few mm cannot be detected due to their inefficient thermal emission. However, the detection of mm-sized particles in PPDs is a clear evidence of grain growth in such disks. Remarkably, mm-sized particles are also preserved from our own PPD, the so-called solar nebula: in primitive meteorites a predominant part of the mass is found in chondrules, mm-sized spherules with ages (determined by radio-isotope dating) placing their formation within the first few million years of the solar nebula. Chondrules were molten by an unknown process and solidified within a short time so that they survived the process of the formation of their parent bodies. Chondrules provide strong evidence that (at least at one location) in the solar nebula, (at least) mm-sized dust aggregates were present.

It is the objective of this article to unveil the growth processes of protoplanetary dust from an experimental as well as from a numerical point of view. We will see which physical interactions dust aggregates undergo in mutual low-velocity collisions and to which growth timescales, mass distributions and dust-aggregate structures this leads. As it will turn out, the wealth of laboratory investigations of the past decade will severely challenge the planetesimal-formation scenario outlined above, particularly by showing that dust aggregates tend to bounce and fragment, rather than stick to one another, in collisions

at velocities $v \gtrsim 1 \mathrm{~m} \mathrm{~s}^{-1}$. It will become clear that collisional sticking alone cannot form $\mathrm{km}$-sized planetesimals.

\section{COLLISION AND AGGREGATION EXPERIMENTS WITH DUST PARTICLES}

It is an experimental challenge to approach the problem of dust growth in PPDs. Experimental parameters, such as dust-particle sizes, dust materials (oxides, metals, silicates, organic material, ices), collision velocities as a function of dust-aggregate size, dust-aggregate morphologies (fractal, porous, compact), the gaseous environment (temperature and pressure) and the charging state of the dust particles should 
match those of the dust in PPDs as closely as possible. It is evident from this list of parameters that one single experiment can never fulfil this. Thus, we chose the approach to piecewise match the dust-growth scenario in PPDs, starting from small dust and low velocities (e.g. Brownian motion) in a more or less self-consistent way all the way to larger aggregates with realistic morphologies and higher collision speeds. The experimental approach is ideally paralleled with numerical simulations of the dust growth, taking into account the experimental results as well as physical models for the static and dynamical interactions between dust particles.

Due to the overwhelming size of the parameter space to be covered by this approach, first results are only available for a limited set of parameters (see Güttler et al. (2010) and Zsom et al. (2010) for details). Fig. 11 schematically shows where we currently stand in terms of completeness. (1) The sizes of protoplanetary dust range from initially $\sim 1 \mu \mathrm{m}$ to planetesimal dimensions of $\gtrsim 1 \mathrm{~km}$, above which gravity becomes the dominant effect in collisions. It is obvious that this size range can never be covered in laboratory experiments. However, for all dust-aggregate sizes treatable in the laboratory ( $\sim 1 \mu \mathrm{m} \ldots 0.1 \mathrm{~m})$, experiments have been performed. (2) The mass ratio between the two dust aggregates that collide in the protoplanetary nebula is also a potential parameter to cover in laboratory experiments. It is not difficult to imagine that an impact of a micrometer-sized dust grain into a cm-sized dust aggregate has a different outcome compared to a collision between two $\mathrm{cm}$-sized aggregates at the same velocity. Currently, laboratory collision experiments with rather similar-sized dust aggregates as well as those with aggregates of very dissimilar sizes have been performed. However, we are yet far away from a complete coverage of this parameter. (3) The completeness of the experimental investigations is best for the collision velocity. The experimental velocities achieved so far reach from $\sim 10^{-4} \mathrm{~m} \mathrm{~s}^{-1}$ for Brownian motion to $\sim 100 \mathrm{~m} \mathrm{~s}^{-1}$ for ballistic impacts. (4) It is also not hard to imagine that the collision velocity influences the morphology of the growing dust aggregates. Extremely low velocities will result in very open-structured, porous dust aggregates, whereas collisions at higher velocities will lead to rather compact dusty bodies. Thus, experiments with arbitrary porosities of the aggregates are desirable. The coverage of the potential porosities is, however, still rather poor. While experiments with compact dust aggregates are easy to perform, there is only a limited number of collision experiments with very porous dust aggregates available. (5) Finally, the question of the dust materials to be used in analog experiments needs to be addressed: from the composition of the major bodies in the Solar System and from calculations of the condensation sequences in protoplanetary disks, four major groups of materials are expected that each dominate their own region within the PPD (Lewis, 1972; Grossman, 1972): in the inner region, where the temperatures are highest, the dust composition is dominated by refractory materials, like oxides or metals; further out, the less refractory silicates condense, followed by the condensation of organic materials and ices in the outer regions of the PPD. Most of the laboratory experiments on protoplanetary dust agglomeration have so far been performed with silicates and only very few dealt with the other protoplanetary dust materials.

The most complete set of experimental investigations on the collision and growth behavior of protoplanetary dust has been using $\mathrm{SiO}_{2}$ as the dust material, with particle sizes around $1 \mu \mathrm{m}$ (see Blum \& Wurm (2008) for details). A large subset of these experiments used idealized dust particles, consisting of monodisperse, spherical $\mathrm{SiO}_{2}$ grains of $0.75 \mu$ m radius (Güttler et al., 2010). Before we review these experiments and the modeling approach for dust aggregates, we have to address two basic questions concerning individual dust particles:

1. Why do individual dust particles adhere to one another?

The formation of dust aggregates in the terrestrial-planet region of PPDs most likely happens in a charge-free environment, the so-called MRI-dead zone (Gammie, 1996; Terquem, 2008). However, triboelectric charging in mutual collisions may provide a source for enhanced stickiness of small aggregates (Poppe et al., 2000b; Marshall et al., 2005) but is unlikely to be responsible for the overall growth of protoplanetary dust. Thus, we can assume that dust particles are basically uncharged so that Coulomb forces cannot be responsible for the 'stickiness' of the dust. In the absence of free charge carriers, dust particles do only interact through van der Waals forces, i.e. stochastic dipole interactions, leading to extremely small and short-ranged cohesion forces. Heim et al. (1999) used 


\begin{tabular}{|c|c|c|c|}
\hline $1 \mu \mathrm{m}$ & $1 \mathrm{~mm}$ & $1 \mathrm{~m}$ & $1 \mathrm{~km}$ \\
\hline \multicolumn{4}{|c|}{ Mass ratios of projectile and target: } \\
\hline 0 & & & 1 \\
\hline \multicolumn{4}{|c|}{ Collision velocities of dust aggregates: } \\
\hline $10^{-4} \mathrm{~m} / \mathrm{s}$ & $10^{-2} \mathrm{~m} / \mathrm{s}$ & $1 \mathrm{~m} / \mathrm{s}$ & $100 \mathrm{~m} / \mathrm{s}$ \\
\hline \multicolumn{4}{|c|}{ Porosities of dust aggregates: } \\
\hline compact & & & porous \\
\hline \multicolumn{4}{|c|}{ Dust materials and temperatures: } \\
\hline $\begin{array}{c}\text { oxides/metals } \\
>1000 \mathrm{~K}\end{array}$ & $\begin{array}{l}\text { silicates } \\
\sim 300 \mathrm{~K}\end{array}$ & $\begin{array}{l}\text { organics } \\
\sim 200 \mathrm{~K}\end{array}$ & $\begin{array}{c}\text { ices } \\
\sim 100 \mathrm{~K}\end{array}$ \\
\hline
\end{tabular}

Fig. 1 Schematic illustration of the parameter space of dust-aggregate collision experiments simulating protoplanetary dust evolution. The red-shaded areas have been covered by experiments, whereas the white regions are (yet) unexplored.

an atomic force microscope, to which they glued individual spherical $\mathrm{SiO}_{2}$ particles with different diameters, and measured the separation forces between these particles and other spherical $\mathrm{SiO}_{2}$ particles, which were glued on a flat substrate. Thus, they were able to confirm the predicted proportionality between binding force and particle radius (Johnson et al., 1971; Derjaguin et al., 1975). Heim et al. (1999) derived typical binding forces of $\sim 10^{-7} \mathrm{~N}$ for micrometer-sized particles.

2. At which impact velocities do individual dust particles stick upon a collision?

Poppe et al. (2000a) performed impact experiments of the same type of micrometer-sized, spherical $\mathrm{SiO}_{2}$ particles onto flat $\mathrm{SiO}_{2}$ substrates. They used a cogwheel dust deagglomerator, which is able to separate the individual dust particles of a powder sample and to accelerate the dust grains in a jet-like fashion (Poppe et al., 1997). The impacts of the dust grains onto the targets were observed with a high-resolution long-distance microscope with a pulsed-laser illumination so that the particle trajectories before and after impact could be determined. Poppe et al. (2000a) found a rather sharp transition from sticking (for $v<v_{\mathrm{c}}$ ) to bouncing (for $v>v_{\mathrm{c}}$ ) at a velocity of $v_{\mathrm{c}} \approx 1 \mathrm{~m} \mathrm{~s}^{-1}$. This threshold velocity increased with decreasing particle size.

Knowing the adhesion and collision properties of the individual $\mathrm{SiO}_{2}$ monomer, we can now concentrate on the growth and collision behavior of dust aggregates. Güttler et al. (2010) reviewed a set of 19 different experiments, which all deal with the evolution of protoplanetary dust aggregates. On top of that, a few new experiments were launched in our laboratory in the past months. Fig. 2] shows the masses of the dust aggregates and the collision velocities of these experiments. In the following, we will consider some of these experiments in more detail. One should bear in mind that - although the experimental coverage of dust-aggregate masses and collision velocities is quite satisfactory (see Fig. 1) - the two-dimensional parameter space shows considerable regions that are relevant to protoplanetary dust growth and are not covered by experiments(see Fig. 2).

The experiments dealing with the initial stage of dust agglomeration in PPDs used homogeneous dispersions of individual micrometer-sized dust particles and observed the agglomeration in lowvelocity collisions. From the work of Poppe et al. (2000a) it is clear that the collision velocities have to be below $1 \mathrm{~m} \mathrm{~s}^{-1}$ so that the sticking probability is close to unity. As the sources for the collision of small particles in PPDs are mainly Brownian motion, relative drift motions, and gas turbulence, experiments concentrated on these effects to observe the onset of agglomeration (Blum et al., 2000; Krause \& Blum, 2004; Wurm \& Blum, 1998; Blum et al., 1998). All these experiments found consis- 


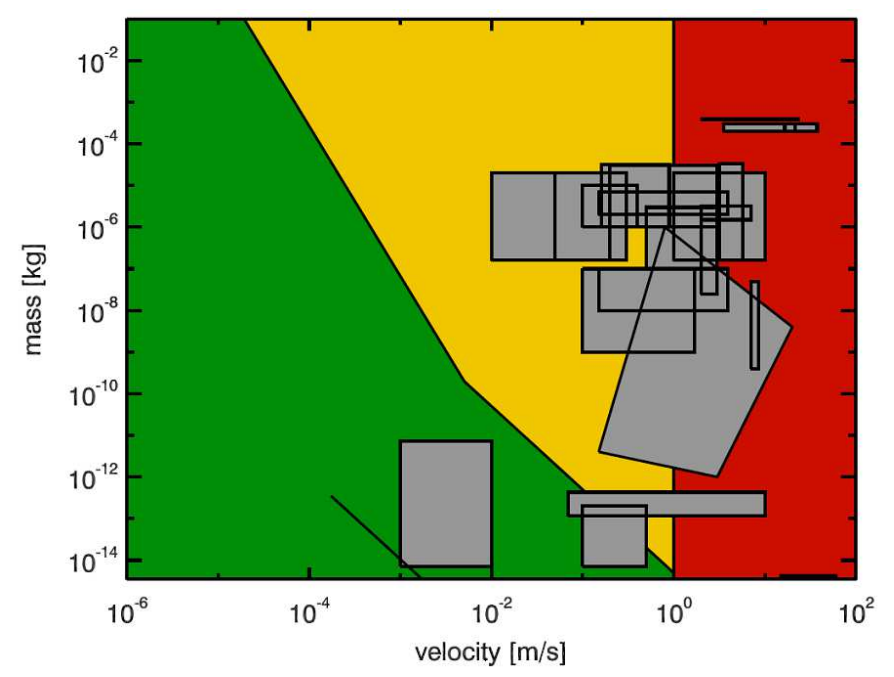

Fig. 2 Dust-aggregate masses and collision velocities of the existing dust-growth experiments. Overplotted are the regions in which sticking (green), bouncing (yellow), and fragmentation (red) are expected. Data taken from Güttler et al. (2010) with augmentation by our latest laboratory experiments.

tently and in agreement with numerical simulations (Kempf et al., 1999; Paszun \& Dominik, 2006) that dust agglomeration in PPDs starts with a fractal growth regime, in which the colliding dust particles stick at the first contact (hit-and-stick growth). The main characteristics of this growth regime are (1) the fractal nature of the growing dust aggregates with a mass $(m)-$ size $(s)$ relation $m \propto s^{D}$, with $D<2$ being their fractal dimension, (2) a quasi-monodisperse mass distribution at any given time $t$, and (3) a power-law temporal growth of the mean mass $<m>\sim t^{2}$ (see Blum (2006) for details). For aggregate sizes exceeding $\sim 100 \mu \mathrm{m}$, Brownian motion becomes slower than the drift motion so that the collision energy increases with increasing aggregate masses. If this energy exceeds a threshold above which frictional forces are no longer sufficient to allow for a hit-and-stick behavior, the dust aggregates are compacted upon collision (Dominik \& Tielens, 1997; Blum \& Wurm, 2000; Wada et al., 2007, 2008, 2009). Thus, it is clear that the 'compactness' or 'fluffiness' of a dust aggregate is an important parameter in the evolution of protoplanetary dust. We describe this 'compactness' or 'fluffiness' either by the porosity of the aggregate (see Fig. 1) or by the enlargement parameter $\Psi=V / V_{\mathrm{c}}$, where $V$ and $V_{\text {c }}$ describe the actual volume and the compact volume of a dust aggregate. The latter is related to the mass of the aggregate through $V_{\mathrm{c}}=\mathrm{m} / \rho_{0}$, with $\rho_{0}$ being the mass density of the monomer grains. It can easily be imagined that it becomes increasingly difficult to perform laboratory experiments with higher and higher porosities. 'Natural' dust aggregates, as they occur in powder samples have porosities of typically $60 \%$ (i.e. enlargement parameters of $\Psi=2.5$ ). Denser aggregates (down to enlargement parameters of $\Psi=1.7$ ) can be manufactured by compressing a dust sample; looser (but still coherent) aggregates can be made by the process of random ballistic deposition (Blum \& Schräpler, 2004), in which individual micrometer-sized dust particles are added one-by-one in a hit-and-stick fashion to grow macroscopic bodies. Random ballistic deposition leads to porosities of $85 \%$ (i.e. to enlargement parameters of $\Psi=6.7$ ) for monodisperse spherical dust particles. Recipes for the manufacturing of large dust aggregates with higher porosities have not been published, although the models for the enlargement parameter of protoplanetary dust aggregates predict values of to $\Psi=15-35$ (Zsom et al., 2010, see also Fig.6p in Sect. 44. 
Experiments on the collisional behavior of dust aggregates with rather high porosities $(\Psi=6.7$, manufactured by the random ballistic deposition process) were performed by Langkowski et al. (2008) and Salter et al. (2009). In combination with the experiments using fractal dust aggregates (Blum et al., 2000; Krause \& Blum, 2004; Wurm \& Blum, 1998; Blum et al., 1998) and those with dust aggregates having low porosities (see Güttler et al. (2010) for details), the emergent picture is that three different collisional outcomes can be distinguished: (1) sticking, in which the mass of at least the larger of the two colliding dust aggregates is increased, (2) bouncing, in which the mass of the interacting bodies is basically unchanged, and (3) fragmentation, in which the mass of the impacting dust aggregates is reduced. Fig. 3]shows experimental examples for sticking, bouncing, and fragmentation. Counter-intuitively, fragmentation can - under certain circumstances - also lead to a mass increase of one (generally the larger) of the dust aggregates (Wurm et al., 2005): if a (smaller) projectile of arbitrary porosity impacts a (larger) target aggregate, which is compact (i.e. having enlargement parameters of $\Psi \lesssim 2$ ), at velocities exceeding the fragmentation threshold (i.e. for $v>1 \mathrm{~m} \mathrm{~s}^{-1}$ ), part of the projectile aggregate (typically a few ten percent of its mass) sticks to the target, thus enhancing the mass of the larger collision partner.

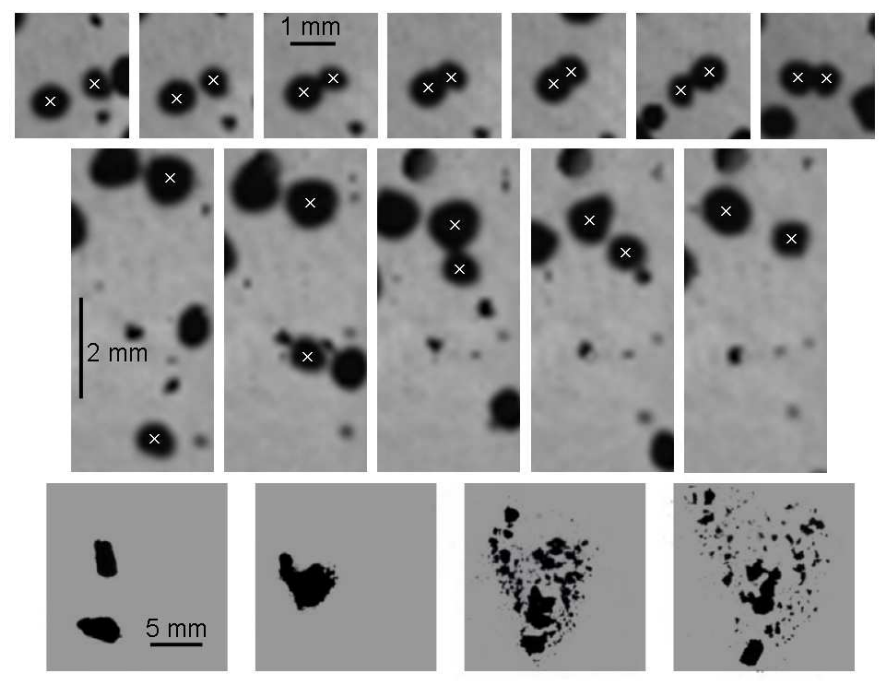

Fig. 3 Examples of laboratory collision experiments between fluffy $\mathrm{SiO}_{2}$ aggregates, which resulted in sticking (top), bouncing (center), and fragmentation (bottom). The collision velocities were $0.004 \mathrm{~m} \mathrm{~s}^{-1}$ (sticking), $0.16 \mathrm{~m} \mathrm{~s}^{-1}$ (bouncing), and $5.1 \mathrm{~m} \mathrm{~s}^{-1}$ (fragmentation), respectively. All experiments were performed under microgravity conditions. For clarity, the colliding dust aggregates in the sticking and bouncing case are marked by an $\mathrm{x}$. The time interval between the first five images in the sticking case is $54 \mathrm{~ms}$, the last three images have time intervals of $850 \mathrm{~ms}$ between them to proof the durability of the adhesion; the time interval between the images in the bouncing case is 14 $\mathrm{ms}$; the time interval between the first two images and the last two images in the case of fragmentation is 1 ms, respectively.

A thorough look at the published protoplanetary-dust experiments reveals that yet another parameter is required to describe the collisional physics of fluffy dust aggregates, i.e. the mass ratio of the colliding 
dust aggregates. Experiments by Langkowski et al. (2008), who observed the impacts of mm-sized highporosity dust aggregates into $\mathrm{cm}$-sized high-porosity target aggregates at various velocities, showed that for colliding dust aggregates with the same porosity but very different sizes the smaller (projectile) aggregate gets buried in the larger (target) aggregate for $v \gtrsim 1 \mathrm{~m} \mathrm{~s}^{-1}$. On the other hand, if, for the identical dust-aggregate morphologies and porosities, the target size is reduced to the projectile size, the experiments show that both aggregates fragment for collision velocities $v \gtrsim 1 \mathrm{~m} \mathrm{~s}^{-1}$ (see Fig. 3. bottom). This means that there is growth for projectile-target collisions, whereas the opposite (i.e. fragmentation) happens if both aggregates are similar in size. For a realistic collision model of protoplanetary dust aggregates it is therefore mandatory to take into account four parameters:

1. the collision velocity,

2. the mass of the projectile aggregate, i.e. the aggregate with the smaller mass,

3 . the mass ratio between the two colliding aggregates,

4. the porosity of the aggregates, i.e. their enlargement parameter.

Thus, for a deeper understanding of the evolution of protoplanetary dust aggregates, a full coverage of this four-dimensional parameter space (for a variety of realistic dust materials) is required. In the following Section, we will describe the main characteristics of our collision model, which takes into account this multi-parameter space. For details, refer to Güttler et al. (2010).

\section{A NEW DUST-AGGREGATE COLLISION MODEL}

To be able to predict the temporal evolution of protoplanetary dust aggregates, a physical model on the collision behavior of arbitrary dusty bodies is mandatory. As we have seen above, a full four-dimensional treatment of the parameter space (mass, mass ratio, porosity, and collision velocity of the dust aggregates) is, however, not yet possible because the coverage of the mass ratio and enlargement parameter is far from being complete. Thus, we decided to treat these two parameters in a binary way in our model (Güttler et al., 2010). We describe dust aggregates either as 'porous' (p) or as 'compact' (c). Without better knowledge, we set the threshold between the two regimes at an enlargement parameter of $\Psi=2.5$ (i.e. a porosity of $60 \%$ ), i.e. all aggregates with $\Psi>2.5$ are 'porous' and those with $\Psi<2.5$ are considered 'compact'. In a similar way, the mass ratio of the colliding aggregate pair is parameterized: for mass ratios between the more and less massive dust aggregate $r<100$, we consider both aggregates to be of equal size; for $r>100$, we treat the collision as an impact of a projectile into an infinitely large target aggregate. Under the assumption of similar porosity of the two colliding dust aggregates, the threshold mass ratio of $r=100$ corresponds to a size ratio of 4.6. With this simplification, the following eight collision types are possible and are independently treated in our collision model: 'pp', 'pP', 'pc', 'pC', 'cp', 'cP', 'cc', and 'cC'. Here, two small letters characterize a collision with $r<100$, whereas a combination of a small and a capital letter depicts a projectile-target collision. Please mind that the 'pc' combination differs from 'cp' in such a way that in 'pc' the (slightly) less-massive of the two colliding aggregates is porous, whereas in the 'cp' case it is compact.

The laboratory experiments have shown that a simple distinction between sticking, bouncing, and fragmentation is insufficient to describe the full suite of possible outcomes of inter-aggregate collisions. Taking into account all cases seen in laboratory experiments, our collision model was generalized and distinguishes between four different types of sticking (S1-S4), in which the more massive of the collision partners gains mass, two types of bouncing (B1-B2), and three types of fragmentation (S1-S3), in which the more massive aggregates loses mass. Fig. [4 shows pictorial representations of these nine collision types.

We now briefly describe the main physical effects leading to the various collision types. The interested reader is referred to Güttler et al. (2010) for a detailed description and the complete set of formulae.

- S1: hit-and-stick collisions.

As described above, the two colliding dust aggregates stick at their first point of contact. This growth 


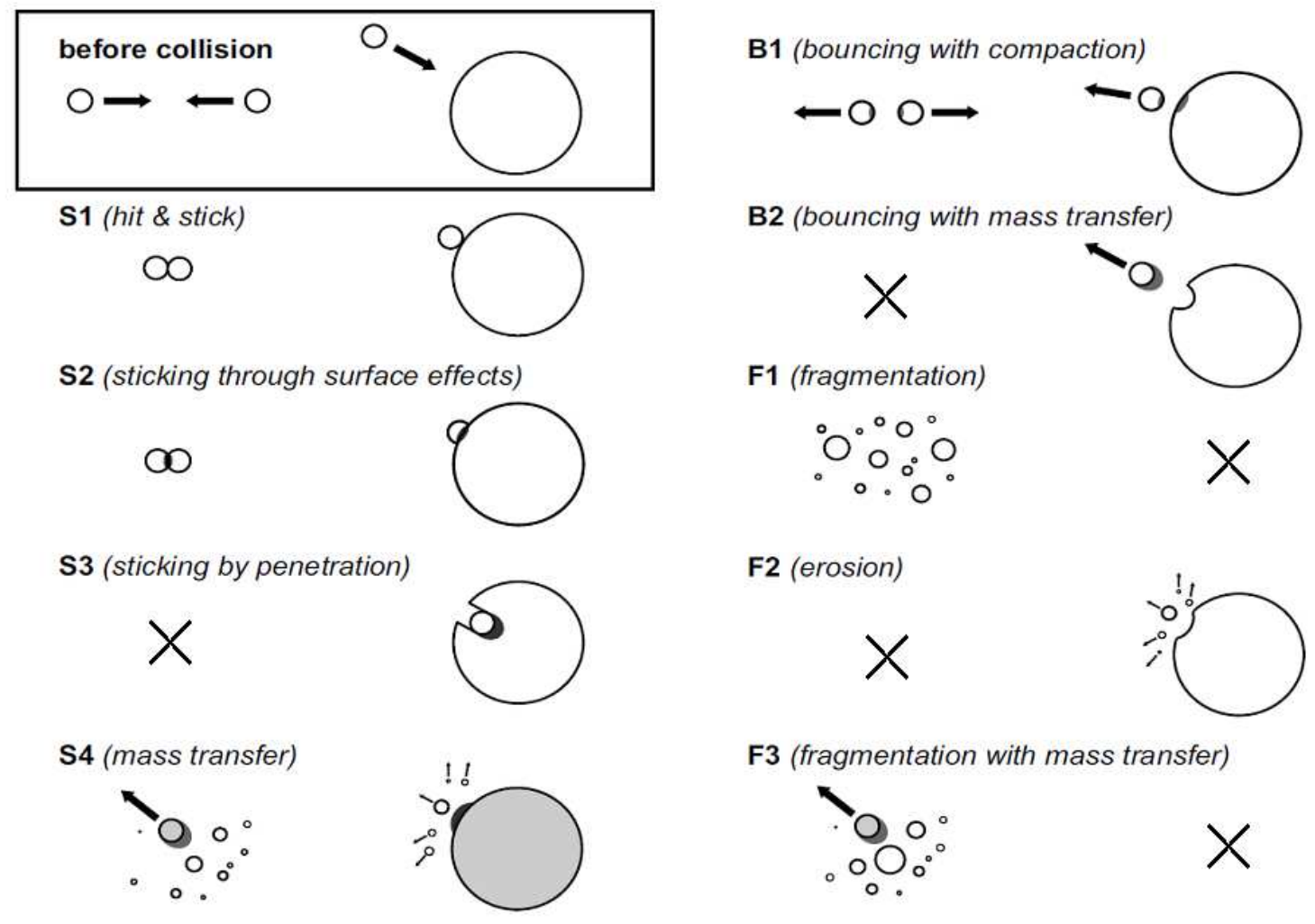

Fig. 4 Pictorial representations of possible outcomes in protoplanetary dust-aggregate collisions (taken from Güttler et al. (2010)). On the respective top parts, collisions between similar-sized aggregates $r<100$ are depicted, whereas the bottom parts show collisions of the projectile-target type $(r>100)$. Gray shading denotes that this particular type of collision happens for compact particles $(P s i<2.5)$ only. The collisional outcomes distinguish between sticking (S1-S4), bouncing (B1-B2), and fragmenting (F1-F3) collisions (see Sect. 3 for details).

type leads to fractal dust aggregates and is limited by the threshold velocity for compaction as an upper velocity limit for S1. The experimental evidence for S1 can be found in Blum et al. (2000); Krause \& Blum (2004); Wurm \& Blum (1998); Blum et al. (1998).

- S2: sticking through surface effects.

For velocities above the S1 limit, dust aggregates can in principle still stick upon a collision if they are able to dissipate sufficient energy and if the contact surface is large enough. Collisional compaction is a process that does both, i.e. it increases the contact surface and is mainly responsible for the dissipation of kinetic energy. Using a Hertzian model for the deformation of fluffy dust aggregates, which was 'calibrated' with the experimental findings, we derived upper limits for dustaggregate sticking. Fig. 3 (top) shows an example for S2.

- S3: sticking by penetration.

If the collision velocities are above the S2 limit, dust aggregates can still gain mass. Process S3 is one method by which this is possible: in the case of projectile-target collisions and porous tar- 
gets, the projectile aggregate does not fragment upon impact but is - above a threshold velocity embedded in the target aggregate, leading to a mass gain of the target (Langkowski et al., 2008).

- S4: sticking through mass transfer.

If, on the other hand, the larger of the two projectiles is compact and the less massive aggregate is porous, and if the collision velocity is above the $\mathrm{S} 2$ limit, the porous aggregate is likely to fragment in the collision. It was observed in experiments (Wurm et al., 2005) that this type of collision results in the attachment of part of the fluffy aggregate's mass to the compact particle.

- B1: bouncing with compaction.

In those cases, in which the collision velocity is above the $\mathrm{S} 2$ threshold and below the threshold for fragmentation or sticking by penetration, the aggregates bounce off. Previous experiments had shown that - even in the case of bouncing - a substantial part of the kinetic energy is dissipated, typically between $80 \%$ and $95 \%$ (Blum \& Münch, 1993). Further experiments by Weidling et al. (2009) showed that the energy is used to restructure the aggregates close to the surface, which leads to local compaction. Thus, repetitive bouncing is a means to convert a 'porous' into a 'compact' dust aggregate. When, after repeated compaction processes, the dust aggregates are sufficiently dense, they might also fragment at velocities below the threshold of process F1 with a small likelihood.

- B2: bouncing with mass transfer.

Bouncing can also be accompanied by a transfer of mass for projectile-target collision between porous dust aggregates. Langkowski et al. (2008) found that this mass transfer always leads to a mass increase of the (smaller) projectile and never of the (larger) target. In B2 collisions, the projectile aggregates typically doubles its mass.

- F1: fragmentation.

At sufficiently high velocities, the internal strength of the dust aggregates is not sufficient to withstand the impact stresses so that the dust aggregates break into smaller fragments. The fragment masses follow a velocity-independent power law, with the largest fragment being a function of impact speed (Blum \& Münch, 1993; Güttler et al., 2010). For the micrometer-sized spherical $\mathrm{SiO}_{2}$ particles used in most of our experiments, the fragmentation boundary is at $v_{\mathrm{c}} \approx 1 \mathrm{~m} \mathrm{~s}^{-1}$, remarkably close to the threshold velocity for sticking of the monomer grains (see Sect.2).

- F2: erosion.

Very small dust particles (e.g. monomer grains) can erode the surface of a much larger dust aggregate. This process initially leads to the mass loss of the porous target aggregate but also compacts it such that the target aggregates gets passivated against erosion up to velocities of $>10 \mathrm{~m} \mathrm{~s}^{-1}$ (Schräpler \& Blum, 2010).

- F3: fragmentation with mass transfer.

Very similar to process F1 and S4, collisions between similar-sized aggregates lead to the fragmentation of the porous aggregates. If the (slightly) larger of the two aggregates is porous and the smaller aggregate is compact, part of the mass of the porous aggregate is transferred to the compact aggregate so that the larger aggregate loses mass and the smaller one gains mass (Güttler et al., 2010). We count this as fragmentation, as the more massive object is fragmented.

It must be emphasized again that the experimental coverage of the parameter space is quite sparse so that a complete collision model has to rely on extrapolations from and interpolations between experimental data points. On top of that, the collision model requires quite a large number of physical parameters as input values (see Table 2 in Güttler et al. (2010)). We carefully measured or estimated these values to complete the model. For dust aggregates consisting of monodisperse, spherical $\mathrm{SiO}_{2}$ particles with $0.75 \mu \mathrm{m}$ radius, our model is shown in Fig. 5. Each panel shows the mass-velocity parameter space for one of the eight combinations of porous/compact and equal-sized/different-sized aggregate collisions. Green color denotes the sticking (S1-S4) processes (mass gain), yellow color represents bouncing (B1-B2), and red stands for the fragmentation cases F1-F3 (mass loss). The collision model does not only predict the qualitative outcome of individual collisions between dust aggregates at different velocities, but also the impact of the collision on the morphology of the collision product. This encompasses (i) for sticking collisions, a recipe for the porosity of the new agglomerate, which can be 
higher (e.g. process S1) or lower (e.g. process S3) than that of the colliding aggregates, (ii) for bouncing collisions, the amount of compaction, (iii) for fragmenting collisions, the size distribution of fragments and the mass of the largest fragment, and (iv) for all collision types the amount of mass transfer between the aggregates. Before we get to the implications of our dust-collision model for the evolution of dust aggregates in PPDs, we take another look at Fig. 5 and try to find out which path the growth process can take. As stated in Sect. 1, the mean collision velocity of dust aggregates in PPDs increases with increasing aggregate size between a few micrometers and one meter in size. Hence, the general growth path must somehow proceed from the lower left to the upper right in Fig. 5 In all but one panels of Fig. 5, this growth trajectory leads through bouncing or fragmentation terrain. The only possible way towards larger dust aggregates can be found in the 'pP' panel, i.e. for collisions between porous dust aggregates of (very) different sizes. The responsible growth process is then $\mathrm{S} 2$, in which the fluffy dust aggregates undergo some compaction during the collision so that the contact area increases, which leads to sticking. As this process is rather well characterized by the experimental results of Langkowski et al. (2008), we can have some confidence that a dust evolution through this growth path can be realistically modelled.

\section{DUST EVOLUTION IN PROTOPLANETARY DISKS}

With the 'complete' dust-aggregate collision model described in Sect. 3, it is possible to predict the collisional evolution of protoplanetary dust aggregates in any accretion-disk model. However, due to the complexity of the collision model, solutions of rate equations are too multi-dimensional to allow sensible results. We chose the Monte-Carlo method proposed by Zsom \& Dullemond (2008) as the solver to the problem of dust evolution in PPDs, using three different PPD models at three different $\alpha$ values each (see Zsom et al. (2010) for the details). As a start and due to the considerable requirements in computational resources, we ran a local simulation, addressing the dust-aggregate evolution at 1 AU from the central solar-type star and in the midplane of the PPD. As relative velocities between the dust aggregates we considered Brownian motion, radial drift and gas turbulence, the latter following the prescription of Ormel \& Cuzzi (2007). Of the three PPD models, the MMSN model resulted in the largest achieved dust aggregates so that we will here discuss only the results of our simulations of dustaggregate evolution in the MMSN model. The main results of the study by Zsom et al. (2010) can be seen in Fig. 6), where in the left panel the temporal evolution of the aggregate masses and in the right panel the corresponding enlargement parameters are shown. The following main characteristics of the dust growth in PPDs must be emphasized:

- From the nine physical processes, which constitute or collision model (see Fig. 5]and Sect. 3), only four are entirely responsible for the grain evolution in the MMSN model. These are S1 (hit-and-stick growth), S2 (sticking through surface effects), B1 (bouncing with compaction), and B2 (bouncing with mass transfer).

- Grain growth is initially dominated by hit-and-stick collisions, in which fractal dust aggregates with $\sim 10^{4}$ monomer grains form within a few hundred years. After that, hit-and-stick (S1) and sticking with compaction (S2) dominate the growth for a few thousand years, forming high-porosity aggregates (with enlargement parameters of up to $\Psi=15-35$ ) with masses of $\sim 10^{-3} \mathrm{~g}$. A short period of runaway growth around $3 \times 10^{3}$ years then produces fluffy dust aggregates with masses of up to a few grams and with enlargement parameters of $\Psi \approx 10$. After that, the collisions among the dust aggregates are dominated by bouncing with compaction (B1), with minor contributions by bouncing with mass transfer (B2), which leads to a considerable reduction of the porosity. After a few $10^{4}$ years, the dust aggregates are basically compact and cannot grow any further, due to a lack of smaller dust.

- When we take into account that compact dust aggregates can break up with a low probability, even at velocities smaller than the typical fragmentation threshold of $\sim 1 \mathrm{~m} \mathrm{~s}^{-1}$, as suggested by the experiments of Weidling et al. (2009), the maximum aggregate mass decreases to $\sim 10^{-3} \mathrm{~g}$. The timescale of the mass decrease (but not the final mass) depends on the fragmentation probability, which is not very well constrained by the present experiments. 


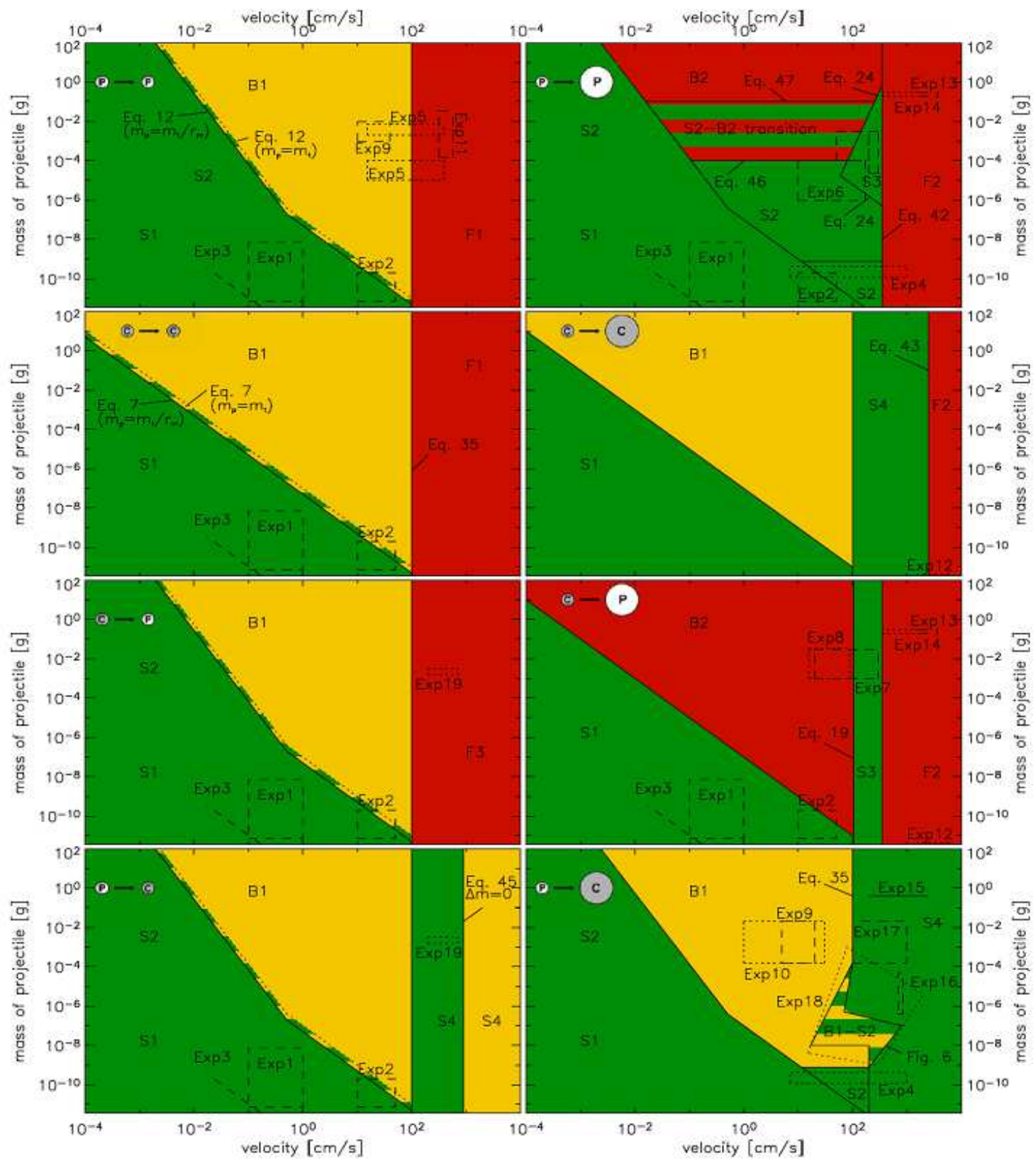

Fig. 5 The collision model for protoplanetary dust aggregates in the mass-velocity parameter space for the eight combinations of aggregate mass ratios and porosities (adapted from Güttler et al. (2010)).

- Aggregate growth stops at the 'bouncing barrier', due to bouncing and not due to fragmentation, so that the maximum aggregate mass found in the model was not achieved by crossing the fragmentation barrier. In fact, fragmentation was never observed for the MMSN model. 
- The dust-aggregate mass distribution in our model is always narrow so that small dust grains are depleted on short timescales. This might seem to contradict the observational evidence which supports retention of small dust particles over an extended period of time (e.g. Furlan et al. (2005); Kessler-Silacci et al. (2006)), but we argue that these observations probe into the atmospheres of the PPDs (where the collision velocities are much higher so that grain growth can be inhibited) whereas our model was applied to the midplane.

- Increasing the collision velocities by applying stronger gas turbulence (with $\alpha$ values ranging from $\alpha=10^{-5}$ to $\alpha=10^{-3}$ ) provides some fragmentation but does not invoke the previously-proposed fragmentation-coagulation cycle (Teiser \& Wurm, 2009), in which the fragmentation events provide sufficiently many small grains for a net growth to occur. The maximum aggregate mass increases sharply from $8 \times 10^{-2} \mathrm{~g}$ at $\alpha=10^{-5}$ to $4 \mathrm{~g}$ at $\alpha=10^{-4}$ and then slightly to $8 \mathrm{~g}$ at $\alpha=10^{-3}$.
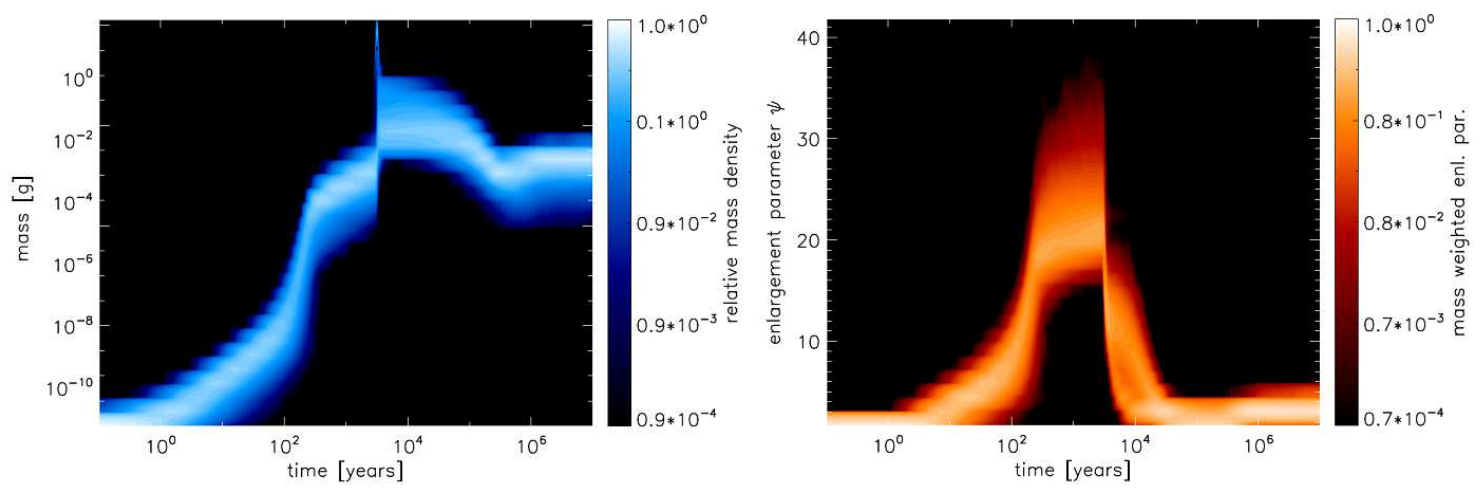

Fig. 6 Results of the Monte-Carlo simulation of the dust evolution in PPDs (adapted from Zsom et al. (2010)). The underlying PPD model was a MMSN model at $1 \mathrm{AU}$ and in the midplane with $\alpha=10^{-4}$. The dust-aggregate collision model was taken from Güttler et al. (2010). The left graph shows the temporal evolution of the mass distribution of the aggregates, whereas the right graph denotes the temporal behavior of the enlargement parameter.

\section{DISCUSSION AND OUTLOOK}

We now have, for the first time, a physical collision model for protoplanetary dust aggregates, covering the full parameter space in aggregate mass and velocity and also dealing with aspects of aggregate porosity and mass ratio of the colliding particles (Sect.3). This model is based upon extensive laboratory work on the collision behavior of dust aggregates. As we have seen in Sect. 4 (see also Fig. 6), MonteCarlo type growth simulations based on this model predict a new 'bouncing barrier' well below the fragmentation threshold. That growth stops due to bouncing is a consequence of our collision model and needs to be critically reviewed. The number of experimental investigations supporting the dustaggregate collision model is not small. However, the coverage of the huge (at least four-dimensional) parameter space in our collision model is far from being complete. In fact, the results of the Monte Carlo simulations show that the pathway of aggregate growth in PPD leads through parameter terrain, which is not at all supported by experiments and which is subject to large extrapolation uncertainties. The strength of a collaborative effort, bringing together expertise from theory and modeling as well as results from laboratory investigations, is that we can now start to investigate these unexplored regions with new and dedicated experiments. Two examples of recently-developed laboratory experiments shall demonstrate this: 
- Collisions between very large and compact dust aggregates.

Our dust-growth model predicts the formation of $\mathrm{cm}$-sized compact aggregates (for $\alpha=10^{-3}$ and $\alpha=10^{-4}$ ) after about $10^{4}$ years (see Fig. 6). The collision velocities of these aggregates in an MMSN model are $\sim 10 \mathrm{~cm} \mathrm{~s}^{-1}$. To investigate these collisions experimentally, we developed a laboratory mini drop tower, in which collisions between two dust aggregates with arbitrary composition in the size range between $\mathrm{mm}$ and $\mathrm{dm}$ and with collision velocities in the range $\sim 1-300 \mathrm{~cm} \mathrm{~s}^{-1}$ can be studied. First experiments with compact $\mathrm{cm}$-sized dust aggregates showed that bouncing is the dominant collisional outcome in the relevant velocity regime around $10 \mathrm{~cm} \mathrm{~s}^{-1}$. We (Beitz, Güttler \& Blum, pers. comm.) found no sticking down to velocities below $1 \mathrm{~cm} \mathrm{~s}^{-1}$ and fragmentation as the dominant process for velocities exceeding $\sim 100 \mathrm{~cm} \mathrm{~s}^{1}$, in full agreement with the model and earlier work (Stewart \& Leinhardt, 2009; Setoh et al., 2010).

- The breakup probability of dust aggregates at velocities below the fragmentation limit.

The previous experiments by Weidling et al. (2009) had shown that with a probability of $\sim 10^{-4}$ dust aggregates can break up in collisions with velocities of $\sim 20 \mathrm{~cm} \mathrm{~s}^{-1}$, which is well below the fragmentation threshold of $100 \mathrm{~cm} \mathrm{~s}^{-1}$. As the final dust-aggregate size strongly depends on this breakup effect, we are interested in determining the breakup probability with a higher accuracy and as a function of collision velocity and aggregate properties. We therefore developed a new experimental setup in which a well-characterized single dust aggregate can undergo repeated collisions with a solid target at a well-defined impact velocity. First experiments have shown that the breakup probability of $\mathrm{mm}$-sized dust aggregates increases considerably when the collision velocity is raised from $20 \mathrm{~cm} \mathrm{~s}^{-1}$ to $50 \mathrm{~cm} \mathrm{~s}^{-1}$ (Rott, Güttler \& Blum, pers. comm.). Once incorporated quantitatively in our collision model, this will surely change the predicted growth behavior of PPD dust aggregates. A detailed investigation is under way.

However, not every parameter combination which is predicted by the Monte-Carlo model is accessible to experiments. Extremely low collision velocities and very high enlargement parameters are basically impossible to achieve in the laboratory and the experimental aggregate-mass range is also finite. A way out of this dilemma is a trustworthy collision model for dust aggregates of arbitrary composition. Molecular dynamics models (see, e.g., Dominik \& Tielens (1997); Wada et al. (2007, 2008, 2009)) are restricted to masses $\lesssim 10^{-6}$ g so that a large part of the parameter space remains uncovered. We have recently published a new Smooth Particle Hydrodynamics (SPH) model for dust-aggregate collisions, based upon earlier work by Sirono (2004) and Schäfer et al. (2007), which might be helpful to fill the remaining parameter space with reliable predictions for the collisional outcomes (Güttler et al., 2009; Geretshauser et al., 2010). Using dedicated laboratory experiments, we were able to calibrate the SPH code for aggregates with an enlargement parameter of $\Psi \approx 7$. Future investigations will concentrate on modelling the collision behavior of dust aggregates with different enlargement parameters.

Our Monte-Carlo simulations indicated that there is no straightforward way for a direct growth of $\mathrm{km}$-sized planetesimals from small dust grains. It is obvious that the bouncing barrier is a stopper for the previous fast growth of protoplanetary dust aggregates. However, these results depend on the validity of both, our dust-aggregate collision model and the model for the collision velocities of the dust particles. The former was developed for refractory silicates and might be more favorable for sticking for icy materials, i.e. in the outer reaches of PPDs (laboratory work on the potentially enhanced stickiness of $\mu \mathrm{m}$-sized ice particles is, however, still lacking). The latter was derived by Weidenschilling \& Cuzzi (1993) and Ormel \& Cuzzi (2007) for a small dust-to-gas ratio in the PPD. Due to the sedimentation of $\mathrm{mm}$ - to $\mathrm{cm}$-sized particles, a dust-dominated sub-disk can form in the midplane of the PPD (if turbulence is negligibly small, i.e. in dead zones of otherwise MRI active PPDs), in which the collision velocities among the dust aggregates can be suppressed. As the dust-aggregate collision model by Güttler et al. (2010) shows (see Fig. 5], an unlimited growth of the protoplanetary dust is possible if there is either a steady source of small, porous dust aggregates, or in collisions between compact aggregates of very different sizes at reduced velocity. Our local aggregate growth model does not predict a sufficiently wide size distribution to support this, but turbulence-driven mixtures of dust aggregates from different regions could help to stimulate a further growth. Work on this is in progress. 
Alternatively, the collective gravitational effect of cm-sized dust aggregates in the dust sub-disk can trigger the growth of macroscopic protoplanetary bodies (Johansen et al., 2007, 2008, 2009). Disk models with sufficiently high global turbulence lead to transient high- and low-pressure zones, which result in a temporal and local enhancement of the number density of dust aggregates. Such dust-enhanced regions rotate with slightly higher velocity and forces the gas to a slightly faster rotation about the central star so that the radial drift rate is locally decreased. This, in turn, means that dust aggregates from outside the dust-enhanced regions can drift into the dust-enhanced clouds so that their mass density is further increased. This 'streaming instability' can ultimately lead to a gravitational instability in the dust, as long as fragmentation events are suppressed (Johansen et al., 2008). The latest simulations predict the formation of planetesimals with sizes of 10-100 km within a few orbital timescales (Johansen et al., 2009), in agreement with the recent result from Morbidelli et al. (2009) that asteroids started around this size. The current minimum size for dust aggregates that can result in gravitational-unstable regions is a few $\mathrm{cm}$, slightly above the maximum size from our simulations. Future investigations will have to show whether this small gap can be closed, particularly as our model shows that breakup of the cm-sized dust ultimately decreases the aggregate sizes to millimeters. Alternatively, Cuzzi et al. (2010) developed a model in which mm-sized particles (Cuzzi et al. (2010) assume chondrules but their physical arguments also hold for equal-mass compact dust aggregates, which are the outcomes of our model) concentrate in low-vorticity regions of a turbulent PPD, in which they can gravitationally interact as an ensemble. Cuzzi et al. (2010) find that for PPD models with higher gas densities, higher dust-to-gas ratios, and shallower radial density gradients than the MMSN model, bodies in the 100-km size range form on timescales compatible with direct age determinations of meteorites and with the initial asteroid sizes derived by Morbidelli et al. (2009).

Such gravitational instability scenarios also have the advantage of avoiding the so-called 'meter-size barrier'. Meter-sized bodies in PPDs have radial drift timescales of the order of 100 years, after which they are lost into the central star. Any model that relies on the direct adhesional growth from dust to planetesimals must pass this barrier within a time span shorter than the lifetime of these bodies. This has been a problem for the previous dust-aggregation model, but is naturally avoided by gravitational instabilities.

It seems as if the two-stage planet-formation paradigm needs some refinement. Aggregation seems to work nicely up to sizes of $\sim 1 \mathrm{~cm}$ but is severely suppressed for larger dust-aggregates, mainly owing to the bouncing barrier. However, due to the formation of a dust sub-disk, the streaming instability can result in gravitationally-unstable regions in the dust, from which asteroid-size bodies can collapse. The final stage in the formation of terrestrial planets is accretion in mutual collisions, which has successfully been modelled in the past.

To assess the validity of such a three-stage planet-formation model, more laboratory experiments, dust-aggregation simulations in at least two spatial dimensions, and detailed investigations of the collisional and gravitational evolution of dense dust-aggregate ensembles are required.

Acknowledgements This work was funded by the German Space Agency (DLR) under grants Nos. 50WM0336, 50WM0636 and 50WM0936, and by the Deutsche Forschungsgemeinschaft (DFG) under grant No. B1298/7-1. I thank Carsten Güttler, Daniel Heißelmann, Christopher Lammel, René Weidling and Andras Zsom for providing me with some of the Figures and Jeff Cuzzi for helpful comments on the manuscript.

\section{References}

Blum, J., \& Wurm, G. 2008, ARA\&A, 46, 21

Blum, J. 2006, Advances in Physics, 55, 881

Blum, J., \& Schräpler, R. 2004, Phys. Rev. Lett., 93, 115503

Blum, J., et al. 2000, Phys. Rev. Lett., 85, 2426

Blum, J., \& Wurm, G. 2000, Icarus, 143, 138

Blum, J., Wurm, G., Poppe, T. \& Heim, L.-O. 1998, Earth, Moon and Planets, 80, 285 
Blum, J., \& Münch, M. 1993, Icarus, 106, 151

Cuzzi, J., Hogan, R., \& Bottke, W. 2010, Icarus, 208, 518

Derjaguin, B. V., Muller, V. M., \& Toporov, Y. P. 1975, J. Colloid Interface Sci., 53, 314

Dominik, C., \& Tielens, A. G. G. M. 1997, ApJ, 480, 647

Dullemond, C. P., Hollenbach, D., Kamp, I., \& D’Alessio, P. 2007, Protostars and Planets V, 555

Furlan, E., et al. 2005, ApJ, 628, L65

Gammie, C. F. 1996, ApJ, 457, 355

Geretshauser, R. J., Speith, R., Güttler, C., Krause, M., \& Blum, J. 2010, A\&A, 513, A58

Grossman, L. 1972, Geochimica et Cosmochimica Acta, 36, 597-619

Güttler, C., Krause, M., Geretshauser, R. J., Speith, R., \& Blum, J. 2009, ApJ, 701, 130

Güttler, C., Blum, J., Zsom, A., Ormel, C. W., \& Dullemond, C. P. 2010, A\&A, 513, A56

Heim, L.-O., Blum, J., Preuss, M., \& Butt, H.-J. 1999, Physical Review Letters, 83, 3328

Johansen, A., Oishi, J. S., Low, M.-M. M., Klahr, H., Henning, T., \& Youdin, A. 2007, Nature, 448, 1022

Johansen, A., Brauer, F., Dullemond, C., Klahr, H., \& Henning, T. 2008, A\&A, 486, 597

Johansen, A., Youdin, A., \& Mac Low, M.-M. 2009, ApJ, 704, L75

Johnson, K. L., Kendall, K., \& Roberts, A. D. 1971, Proc. R. Soc. London A, 324, 301

Kempf, S., Pfalzner, S., \& Henning, T. K. 1999, Icarus, 141, 388

Kessler-Silacci, J., et al. 2006, ApJ, 639, 275

Krause, M., \& Blum, J. 2004, Physical Review Letters, 93, 021103

Langkowski, D., Teiser, J., \& Blum, J. 2008, ApJ675, 764

Lewis, J. 1972, Icarus, 16, 241

Marshall, J., Sauke, T., \& Cuzzi, J. 2005, Geophys. Res. Lett., 32, L11202

Morbidelli, A., Bottke, W. F., Nesvorný, D., \& Levison, H. F. 2009, Icarus, 204, 558

Natta, A., Testi, L., Calvet, N., Henning, T., Waters, R., \& Wilner, D. 2007, Protostars and Planets V, 767

Ormel, C., \& Cuzzi, J. 2007, A\&A, 466, 413

Paszun, D., \& Dominik, C. 2006, Icarus, 182, 274

Poppe, T., Blum, J., \& Henning, T. 2000, ApJ, 533, 454

Poppe, T., Blum, J., \& Henning, T. 2000, ApJ, 533, 472

Poppe, T., Blum, J., \& Henning, T. 1997, Rev. Sci. Instrum., 68, 2529

Salter, D., et al. 2009, Rev. Sci. Instrum., 80, 074501

Schäfer, C., Speith, R., \& Kley, W. 2007, A\&A, 470, 733

Schräpler, R., \& Blum, J. 2010, ApJ, submitted

Setoh, M., Nakamura, A., Michel, P., Hiraoka, K., Yamashita, Y., Hasegawa, S., Onose, N., \& Okudaira, K. 2010,

Icarus, 205, 702

Sirono, S. 2004, Icarus, 167, 431

Stwart, S., \& Leinhardt, Z. 2009, ApJ, 691, L133

Teiser, J., \& Wurm, G. 2009, MNRAS, 393, 1584

Terquem, C. E. J. M. L. J. 2008, ApJ, 689, 532

Wada, K., Tanaka, H., Suyama, T., Kimura, H., \& Yamamoto, T. 2007, ApJ, 661, 320

Wada, K., Tanaka, H., Suyama, T., Kimura, H., \& Yamamoto, T. 2008, ApJ, 677, 1296

Wada, K., Tanaka, H., Suyama, T., Kimura, H., \& Yamamoto, T. 2009, ApJ, 702, 1490

Weidenschilling, S. J. 1977, MNRAS, 180, 57

Weidenschilling, S. J., \& Cuzzi, J. N. 1993, Protostars and Planets III, 1031

Weidling, R., Güttler, C., Blum, J., \& Brauer, F. 2009, ApJ, 696, 2036

Wurm, G., \& Blum, J. 1998, Icarus, 132, 125

Wurm, G., Paraskov, G., \& Krauss, O. 2005, Icarus, 178, 253

Zsom, A., \& Dullemond, C. P. 2008, A\&A, 489, 931

Zsom, A., Ormel, C. W., Güttler, C., Blum, J., \& Dullemond, C. P. 2010, A\&A, 513, A57 